\title{
An Infrared Small Target Detection Algorithm Based on High-speed Local Contrast Method
}

\author{
Zheng Cui, Jingli Yang*, Shouda Jiang, Junbao Li \\ Department of Automatic Testing and Control \\ Harbin Institute of Technology, Harbin 150080, China
}

\begin{abstract}
Small-target detection in infrared imagery with a complex background is always an important task in remote sensing fields. It is important to improve the detection capabilities such as detection rate, false alarm rate,speed, ect. However, current algorithms usually improve one or two of the detection capabilities while sacrificing the other. In this letter, an Infrared (IR) small target detection algorithm with two layers inspired by Human Visual System (HVS) is proposed to balance those detection capabilities. The first layer uses high speed simplified local contrast method to select significant information. And the second layer uses machine learning classifier to separate targets from background clutters. Experimental results show the proposed algorithm pursue good performance in detection rate, false alarm rate and speed simultaneously.
\end{abstract}

Keywords: small target detection, human visual system, high speed local contrast method, machine learning

\section{Introduction}

Infrared(IR) small-target detection plays a critical role in large amounts of practical projects such as infrared warning and defense alertness, in which not only accuracy is needed but also robustness is required[1].

Various algorithms have been developed in the past few decades[2][3][4]. Conventional small target detection methods such as top-hat filter [2], max-mean/max-median filter[3], highpass filters based on LS-SVM[4], nonparametric regression method[5], Bayesian estimation[6] and two-dimension minimum mean square error(TDLMS) method[7] are widely used to reduce the background clutters. Guo[8] proposed a model multi-channel adaptive mixture background model to detect the target. Beyond that, a series of simple and fast algorithm based on Fourier Transform was proposed, such as spectral residual(SR)[9], phase spectrum of quaternion fourier transform(PFT)[10] ,hypercomplex fourier transform(HFT)[11]. With regards to small target detection, frequency domain methods are quite different from other methods. They transform the airspace information to the frequency domain, and testing in the frequency domain. Some researchers use a variety of tracking algorithms to find the target, such as Kalman

${ }^{*}$ Corresponding author.

Email address: 18745136601@163.com (Jingli Yang) 
filtering[12]. In recent years, methods based on the human visual system have been proposed. HVS is a kind of layered image processing system consisting of optical system, retina and visual pathways, which is nonuniform and nonlinear. Researchers found that using HVS to detect target could get favorable results. That is because human vision has selective attention property which can help people search the salient target from complex unknown scene quickly and precisely without being influenced by complex background. For instance, Sungho Kim et al.[13] proposed a novel HVS contrast mechanism based detecting algorithm, which is capable of increasing target intensity as well as suppressing background clutter and noise.

Although numerous methods have been proposed, many of them may fail in certain circumstances[14]. When they were used in surveilling the ground by a helicopter or an unmanned aerial vehicle(uav), the targets have a recognizable shape[15] but a size of small target and easily overlapped by vegetation, roads, rivers, bridges. In view of the above reasons traditional algorithm produce huge false alarm rate in result. algorithms based on machine learning and neural networks are very effective for this kind of problems, but their computation speed can not get guarant. In order to design an appropriate method, a small target detection method inspired by the human visual system(HVS) has been designed in this paper.

\section{Algorithm Inspired by HVS}

\subsection{Two layers Framework}

Here is the proposed framework inspired by HVS with two layers (See Fig1). The basic idea of Hierarchical framework is to ensure that the target is detected by the first layer, even if the background clutters were also included. Then, screen out the background clutters from the detection results of the first layer in the second layer. On the other hand, as a component of low-level artificial vision processing, it facilitates subsequent processing by reducing computational cost, which is a key consideration in real-time applications.

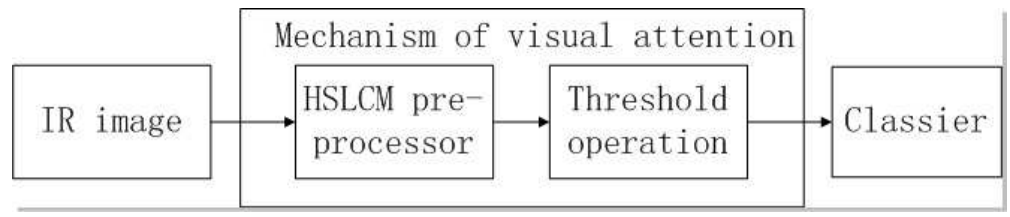

Figure 1: The proposed framework inspired by HVS.

Small infrared target has no texture information or direction information but a higher brightness comparing with the background. That means contrast is the most important quantity encoded in the streams of visual system. With these considerations in mind, we select the contrast of brightness as a standard to decide whether pay attention to the areas or not. There are many methods based on brightness contrast. LCM algorithm is popular because of its high detection rate and lower false detection rate.

After that, some complex processing is performed to extract targets from candidate targets in the second layer. 
In view of background clutters are similar to the infrared targets in the size and shape, it is very difficult for traditional algorithms to distinguish them[16]. The machine learning method, such as support vector machines (SVM)[17] classifier, making use of the statistical features of targets, which will be very effective for this kind of classification problem. Details will be described in section 2.3.

We believe that these two stages would get a better balance algorithm[5].

\subsection{First Layer Processing Method}

The details of HSLCM will be introduced in this section. Here are some basic definitions.

As shown in Fig.2.2, $\mathrm{u}$ denotes the local region, and the area between $\mathrm{u}$ and $\mathrm{v}$ is the local background region. Moreover, the whole image (frame) is denoted by w. In this case, the windows v can move on the w.

Based on the aforementioned preparation, we can find that different image patches of size $\mathrm{v}$ can be obtained by moving the window v on the whole image. Furthermore, u can move on v. Consequently, the obtained image patches can be given by nine cells (see Fig.2.2).

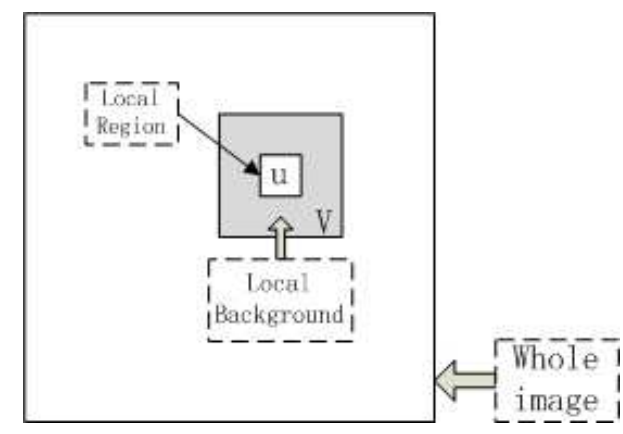

Figure 2: Multiple windows.

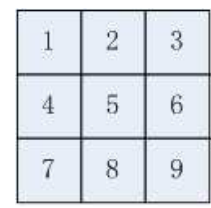

Figure 3: Image patch obtained by sliding window.

Through the analysis of LCM algorithm, we find out that several average operations and calculated pixel by pixel are both time consuming. The saliency c of LCM is defined as follows.

$$
c_{L C M}=\frac{g_{\max }^{2}}{m_{\max }}
$$


$g_{\max }$ expresses the maximum of the gray value of the central cell and $m_{\max }$ express the maximum gray mean of the cells in the local background. In order to increase computation speed of LCM, we try to simplify the model. Like LCM, HSLCM describes a pixel (position) by generating a signal value.

Instead of the maximum gray mean, we use the average of all neighboring cells gray values to be the comparison, which makes the saliency $\mathrm{c}$ as follows.

$$
c_{H S L C M}=\frac{g_{\text {max }}^{2}}{m_{\text {all }}}
$$

$m_{\text {all }}$ can be defined as follows.

$$
m_{\text {all }}=\frac{1}{N} \sum_{i=1, i \neq 5}^{9} \sum_{j=1}^{N} g_{i j}
$$

where $\mathrm{N}$ is the number of the pixels in the $i_{t h}$ cell and $g_{i j}$ is the gray level of the $j_{t h}$ pixel in the $i_{t h}$ cell. Cell 5 is the central cell, so $\mathrm{i}$ is not equal to 5 .

Obviously the new method could be much faster than the original method. Then we analyze amount of calculation of the two algorithms.

Assuming the size of the cell to a $\mathrm{x}$ a and image pixels to N. Each pixel has a $*$ a $* 8$ times of add operation, 8 times of division operation and $\mathrm{a} * \mathrm{a}+7$ times of comparison operations needs to be done for traditional LCM algorithm. But for HSLCM algorithm, only a $* \mathrm{a} * 8$ times of add operation, 1 time of division operation and a $*$ a times of comparison operations needs to be done. Due to the 7 times of division operation and 7 times of comparison operations reduced for each pixel, the new method could be much faster than the original method. Therefore, the amount of calculation for a picture will reduced by $7 * \mathrm{~N}$ times of division operation and $7 * \mathrm{~N}$ times of comparison operations.

HSLCM algorithm also brings some influence to calculation accuracy which will be analyzed from the configuration of the target. Due to the optics point spread function (PSF) of the thermal imaging system at a long distance, there are often two kinds of small target shape, circular and elliptic. For the convenience of analysis, we do some assumptions as the prerequisite. First of all, the small target is assumed to be a 25 pixels (generally considered small target accounts for less than $0.15 \%$ of the total size of the image, our images have $128 \times 128$ pixels)spot. In the ideal case, the size of cell should be the same as the target size.

Average of all gray values in 8 neighboring cells is considered approximate to be background brightness. Refer to the commonly used definition, the contrast of target and background is as follows:

$$
\eta=\frac{G_{t}-G_{b}}{G_{b}}
$$

Where $G_{t}$ is the grayscale average of targets, and $G_{b}$ is the grayscale average of background. So the average of all 8 neighboring cells gray values can be consider as $G_{b}$ and evaluate as $\frac{G_{t}}{1+\eta}$. The contrast ratio of dim small targets and the background is generally considered as 0.15 or less than it. 
Then,we discussed three kinds of circumstances which difference in locations of center pixel relative to a target.

1, The central part is almost all targets(central part of a circular target).

2, Part of the center cell is targets(edge of a circular target).

3 , There is no target in the center cell(outside of a circular target).

Three of the schematic diagram is as follows(see Fig.4).

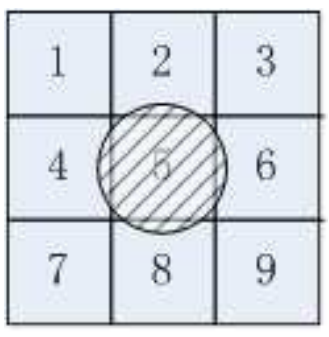

(a)

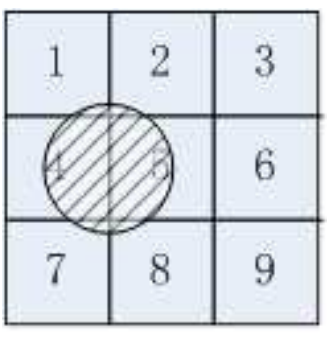

(b)

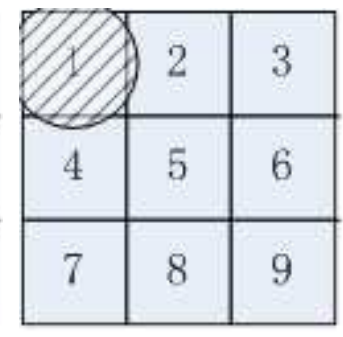

(c)

Figure 4: different parts of a circular target. (a)central part of a circular target. (b)edge of a circular target. (c)outside of a circular target.

Using the new method has no effect on (a).

For circumstance (b), the significant degree of cell 5 is strengthened. Apparently, cell 4 is the brightest in all surrounding cells. So the significant degree of original LCM algorithm is as follows:

$$
c_{L C M} \approx \frac{G_{t}^{2}}{\frac{G_{t}+G_{b}}{2}}=\frac{(1+\eta)^{2} G_{b}^{2}}{\left(1+\frac{\eta}{2}\right) G_{b}}=\frac{2(1+\eta)^{2}}{2+\eta} G_{b}
$$

Based on our assumptions before, the significant degree of HSLCM algorithm is as follows.

$$
c_{H S L C M} \approx \frac{G_{t}^{2}}{G_{b}}=(1+\eta)^{2} G_{b}
$$

$c_{H S L C M} \geq c_{L C M}$ help us to come to a conclusion that the effect of $c_{H S L C M}$ is better than $c_{L C M}$.

In the case of (c), the original method has an inhibiting effect to the significant degree of cell 5, but improved method has not. Here follows our analysis. Obviously, the brighter the around, the stronger the inhibition. Continue to use previous assumptions, the significant degree of original LCM algorithm is as follows.

$$
c_{L C M} \approx \frac{G_{b}^{2}}{G_{t}}=\frac{G_{b}^{2}}{(1+\eta) G_{b}}=\frac{G_{b}}{1+\eta}
$$

The significant degree of SLCM algorithm is as follows.

$$
c_{H S L C M} \approx \frac{G_{b}^{2}}{G_{b}}=G_{b}
$$

$c_{L C M} \geq c_{H S L C M}$ help us to come to a conclusion that the effect of $c_{L C M}$ is better than $c_{H S L C M}$.

Based on the analysis above, we can come to the conclusion that HSLCM has a higher marginal significant degree and also a higher significant degree of the background outside of the target. A higher marginal significant degree 
means the target is more unambiguous, but A higher significant degree of background means oppositely. We can compare the ratio between the two significant degree to do some simple quantitative analysis. Ratios of the two algorithms are as follows.

$$
\begin{gathered}
m_{L C M}=\frac{\frac{2(1+\eta)^{2}}{2+\eta} G_{b}}{\frac{G_{b}}{1+\eta}}=\frac{2(1+\eta)^{3}}{2+\eta} \\
m_{H S L C M}=\frac{(1+\eta)^{2} G_{b}}{G_{b}}=(1+\eta)^{2}
\end{gathered}
$$

$m_{L C M} \geq m_{H S L C M}$ means targets of $c_{L C M}$ are more obvious than $c_{H S L C M}$. Thus, SLCM may be more sensitive to threshold in the following operation.

After analyzing of the above three conditions, we discuss targets with noncircular shape. The small target is assumed to be a spot with a shape of the ellipse. Be similar with the circular target, we discussed four kinds of circumstances (See Fig.5).

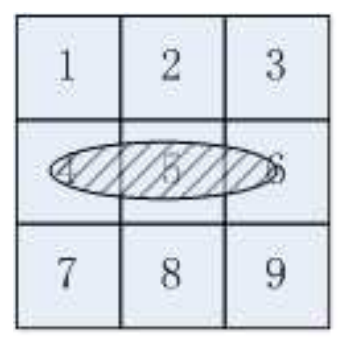

(a)

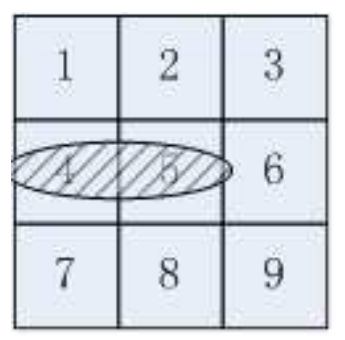

(b)

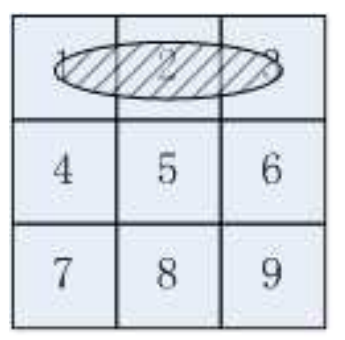

(c)

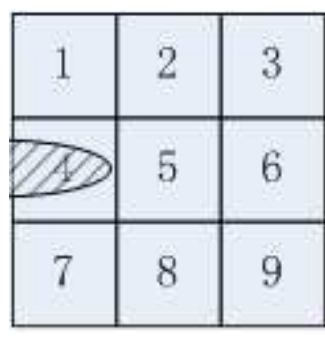

(d)

Figure 5: different parts of a ellipse target. (a)central part of a ellipse target. (b)edge of a ellipse target. (c)outside of a ellipse target's long side.(c)outside of a ellipse target's short side.

Due to the influence of the shape, in situation (a), elliptic targets might have more parts on surrounding cells. Therefore, reference formula 1 , the denominator of $c_{L C M}$ will be affected by the part of the target locate in cell 4 or cell 6.However, reference formula 2 , background brightness would has a more important impact on the denominator of $c_{H S L C M}$. It causes the denominator of $c_{H S L C M}$ smaller than the denominator of $c_{L C M}$ which means $c_{H S L C M} \geq c_{L C M}$.

For circumstance (b), we can get a similar conclusion that the significant degree of LCM algorithm is obviously smaller than the significant degree of HSLCM algorithm. It makes HSLCM easier to enhance the target pixel brightness.

In both case (c) and (d), the denominator of $c_{L C M}$ will be affected by the part of the target. However, the denominator of $c_{H S L C M}$ is almost the same level as background brightness. That means the significant degree of LCM algorithm is smaller than the significant degree of HSLCM algorithm.

The conclusion of ellipse target is similar with the circular target's. however circumstance (b) of ellipse target has a bigger size. That means HSLCM is more suitable for small target with a shape of the ellipse. For target in forward 
looking infrared image as mentioned in introduction, HSLCM has a good adaptability to get the significant map.

After the final significant map be obtained, a threshold operation is used to pick out the most salient points. Next, define the thresholds as followed.

$$
T=\frac{1}{N_{I}} \sum_{j=1}^{N_{I}} S_{j}+k *\left(\operatorname{Max}\left(S_{j}\right)-\frac{1}{N_{I}} \sum_{j=1}^{N_{I}} S_{j}\right)
$$

Where $S_{j}$ represents the saliency value of the $j_{t h}$ pixel,$N_{I}$ is the number of the pixels of the image. k is a parameter from 0 to 1 and be used to control the threshold changing from $\frac{1}{N_{I}} \sum_{j=1}^{N_{I}} S_{j}$ to $\operatorname{Max}\left(S_{j}\right)$. We only deal with those areas above the threshold. Setting threshold can effectively reduce the number of salient areas, thus reduce the amount of calculation.

\subsection{Second Layer Processing Method}

The thought of detection algorithm based on machine learning is converting a target detection problem into a pattern classification problem. The core of this detection algorithm is making full use of prior knowledge.

A SVM is a new machine learning technique developed on the basis of statistical learning theory, and it is the most successful realization of statistical learning theory. It constructs an optimal hyperplane utilizing a small set of vectors near boundary, which can solve small sample learning problems better. Our background of study and application determines our samples can't be comprehensive which means SVM suit our field well.

As a preparation for classified correctly, it is important to obtain training sets by using the statistical features of the small targets. An accurate sample library of the target and the background is the key of the correct classification. Select a sequence of images, using the sequence of the first a few images as the training sample. We use true targets as target samples and use pseudo targets as background samples which belong to the salient regions of the first a few pictures.

Considering the target shape may have a slight change in the process of moving, which because of the change of direction and attitude, we could update the training set to make the classifier more intelligent. This process includes join target and false target of the testing image into the training set respectively.

After preparation, we define the centers of salient areas obtained in the section 2.2 as the suspected target centers and use the SVM classifier to classify these pixels with its neighbors. In our letter, the small target is assumed to be a 25 pixels (generally considered small target accounts for less than $0.15 \%$ of the total size of the image, our images have $128 \times 128$ pixels)spot. Because the small target has a certain shape, it can't be a straight line. Assume a worst case, 25 pixels are divided into two rows. This means that the longest edge of small target is shorter than 13 pixels. So we made sample frame should be at least 13 pixels. However, we don't know the direction of the small target. So we have to use a $16 \times 16$ square.

Due to the SVM classifier can separate target from clutters, the accuracy can be improved. 


\section{EXPERIMENTAL RESULTS AND ANALYSIS}

Experiments on the real IR image sequence have been done. The experimental data including several group of images with a resolution of $(128 \times 128)$, obtained from United States Army Aviation and Missile Command (AMCOM). All experiments were implemented by MATLAB software on a PC with 4-GB memory and 3.2-GHz Intel i5 dual processor. In section 2.1, our algorithm can be divided into two stages. Therefore, experimental results of two stages will be discussed in section 3.1 and section 3.2.

\subsection{HSLCM result}

In section 2.2, small targets with circular and elliptic shapes have been analyzed respectively. Thus, we choose two groups of images(see Fig.6) separately verify these two analysis result. The real targets are marked in two examples.

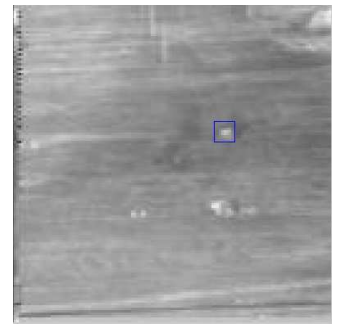

(a)

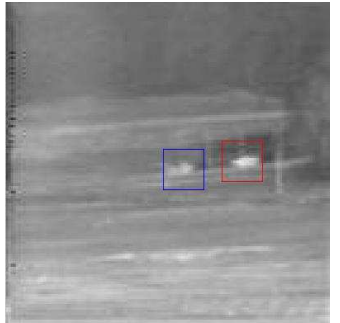

(b)

Figure 6: (a)circular target. (b)elliptic target.

First, we verify the conclusion about circular target. The same image in Fig.6(a) is used to be the example. Here follows the figures of simulation results(see Fig.7 and Fig.8).In these figures, (a) is the saliency map. (b) is the result after the same threshold operation. (c) is the detection result and all the targets are labeled by asterisks.The cell size is set to $(5 \times 5)$. Parameter $\mathrm{k}$ in threshold is set to 0.25 .

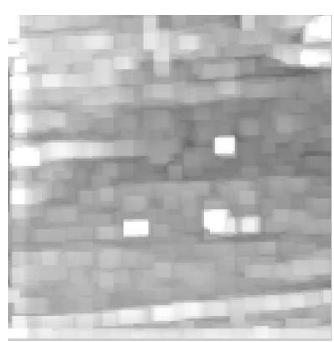

(a)

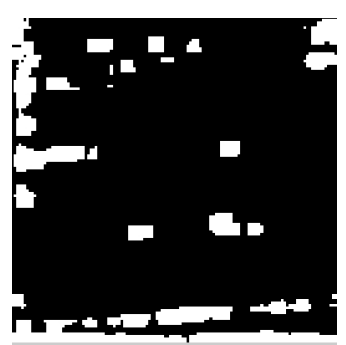

(b)

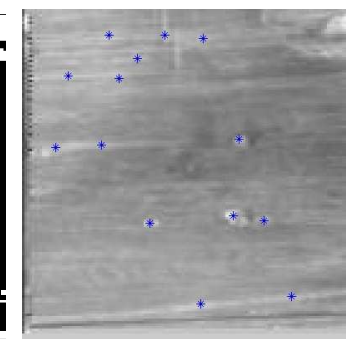

(c)

Figure 7: HSLCM calculation. (a)saliency map. (b)result of threshold operation. (c)detection result.

It can be seen that the effect of HSLCM is very approximate to LCM. 


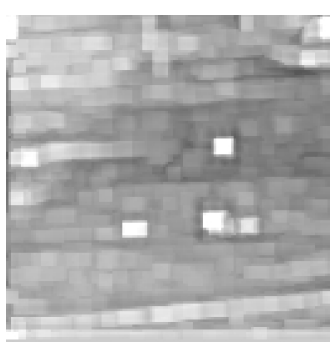

(a)

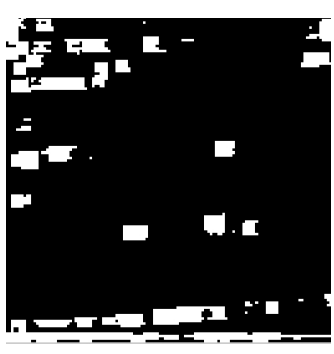

(b)

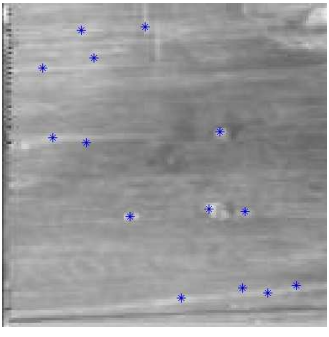

(c)

Figure 8: LCM calculation. (a)saliency map. (b)result of threshold operation. (c)detection result.

To reveal the advantages of the proposed algorithm to the other three algorithms, set algorithms threshold to different values and compare the detection rate [true positive rate (TPR) defined in (12)][18] in Fig.9. Experimental results show that the parameter $\mathrm{k}$ in (8) is suitable to be set from 0.1 to 0.3 in our work. We can get high TRP with all kinds of algorithms by selecting this parameters section so as to meet the requirements.

TPR defined as

$$
T P R=\frac{\text { Quantity of true } T B \text { detected in images }}{\text { Quantity of true TB existing in images }} * 100 \%
$$

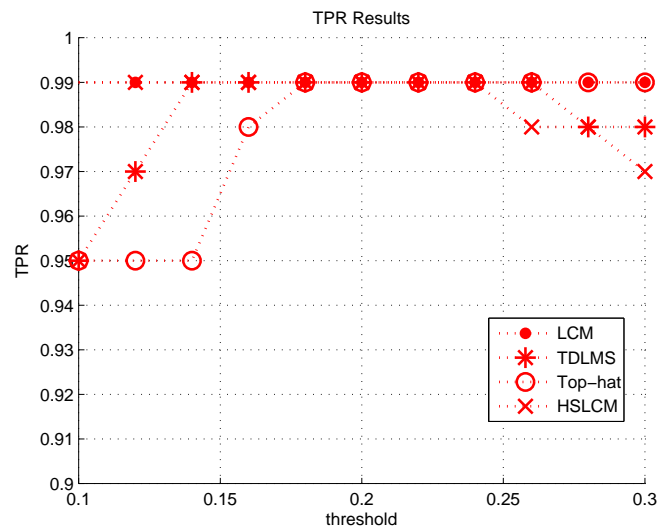

Figure 9: TPR of circular target using four methods.

From the experimental results, we can get the conclusion that HSLCM has almost the same performance with LCM. Although, just like our analysis, HSLCM is sensitive to the threshold, the TPR of it still achieve 99\%. Therefore, for circular target, HSLCM palys a good effect. Our analysis has been affirmed.

Then, we verify the conclusion about elliptic target. The same image in Fig.6(b) is used to be the example. Simulation results are shown in Fig.10 and Fig.11.In these figures,we use a same cell size and parameter k.

We do the same operation as circular target. Experimental results show that the parameter $\mathrm{k}$ in (8) is suitable to be set from 0.1 to 0.3 in our work(See Fig.12). 


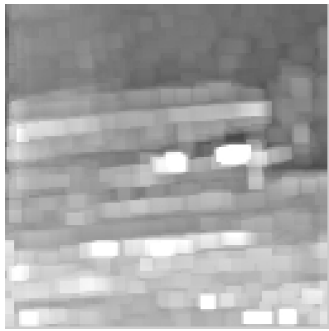

(a)

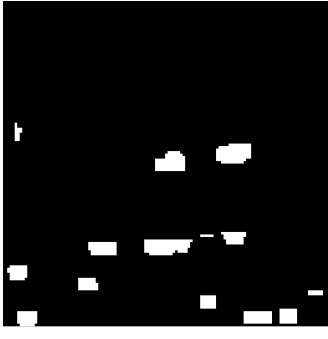

(b)

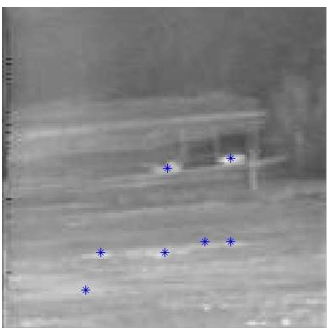

(c)

Figure 10: HSLCM calculation. (a)saliency map. (b)result of threshold operation. (c)detection result.

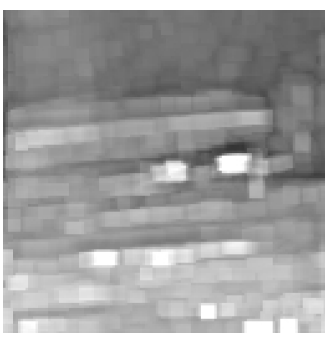

(a)

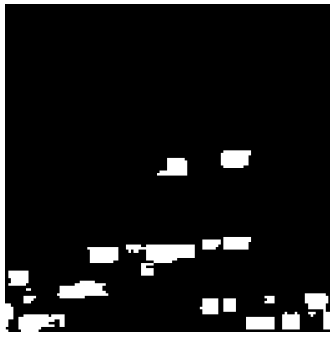

(b)

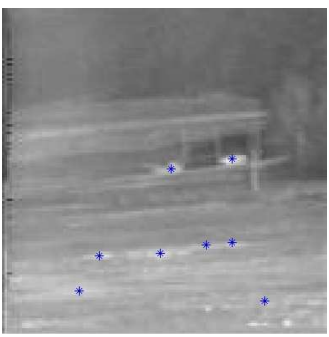

(c)

Figure 11: LCM calculation. (a)saliency map. (b)result of threshold operation. (c)detection result.

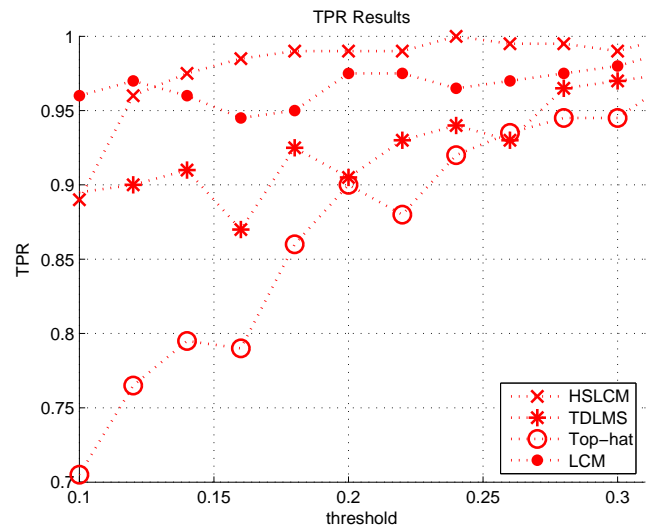

Figure 12: TPR of elliptic target using four methods. 
From the experimental results, we can get the conclusion that HSLCM and LCM can achieve the best performance in TPR.

\section{2. $H S L C M+S V M$ result}

Although the targets are successfully detected, there are many false alarms existed. We hope to reduce the false alarm rate [false positive rate (FPR) defined in (13)] by using the SVM classifier. The penalty factor of SVM classifier is 30. SVM classifier using the gaussian kernel and its parameter is 0.01 . All parameters are selected according to the experience.

FPR defined as

$$
F P R=\frac{\text { Quantity of false TB detected in images }}{\text { Quantity of true TB existing in images }} * 100 \%
$$

Experimental results using the proposed algorithm are shown in Fig.13.

Fig.13(a) is the detection result of HSLCM. Fig.13(b) is the detection result of HSLCM and SVM classifier. Obviously, SVM classifier can effectively distinguish the false target and the real goal.

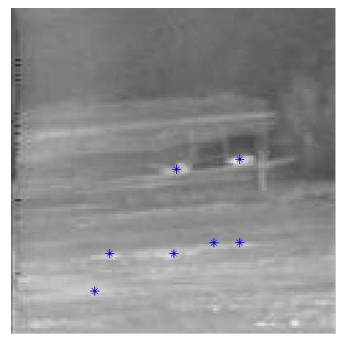

(a)

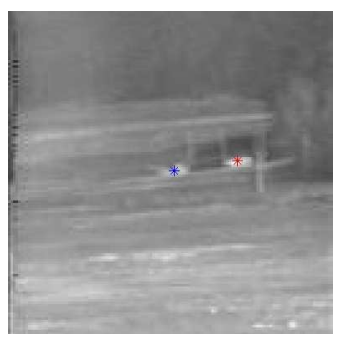

(b)

Figure 13: comparison between detcet results of HSLCM and HSLCM with SVM classifier for elliptic target detection. (a)detcet result of HSLCM. (b)detcet result of SLCM with SVM classifier.

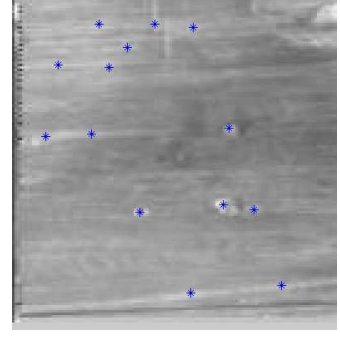

(a)

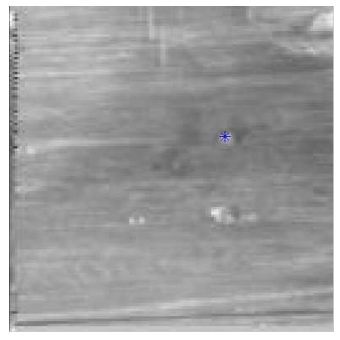

(b)

Figure 14: comparison between detcet results of HSLCM and HSLCM with SVM classifier for circular target detection.(a)detcet result of HSLCM. (b)detcet result of HSLCM with SVM classifier.

We set algorithms threshold to different values and compare false alarm rate of all methods to prove the effectiveness of the introduction of SVM classifier(circular target in Fig.15(a) and elliptic target in Fig.15(b)). We also compare 
detection rate of the two method above to show the influence of the introduction of SVM classifier to TPR(circular target in Fig.16(a) and elliptic target in Fig.16(b))

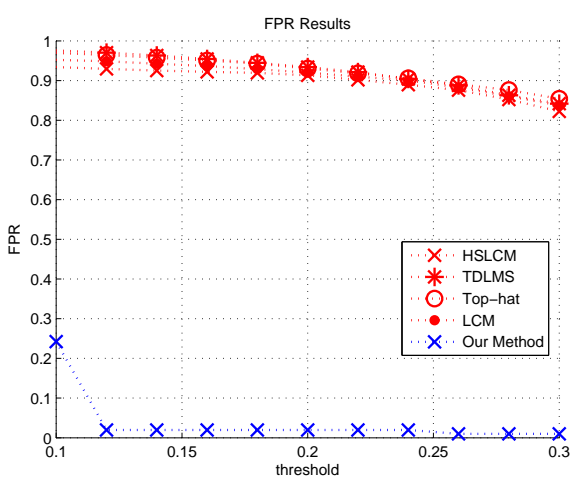

(a)

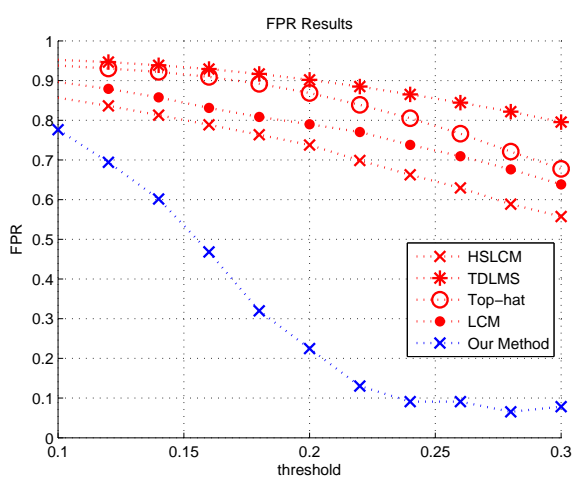

(b)

Figure 15: comparison of several methods' FPR.(a)FPR for circular target. (b)FPR for elliptic target.

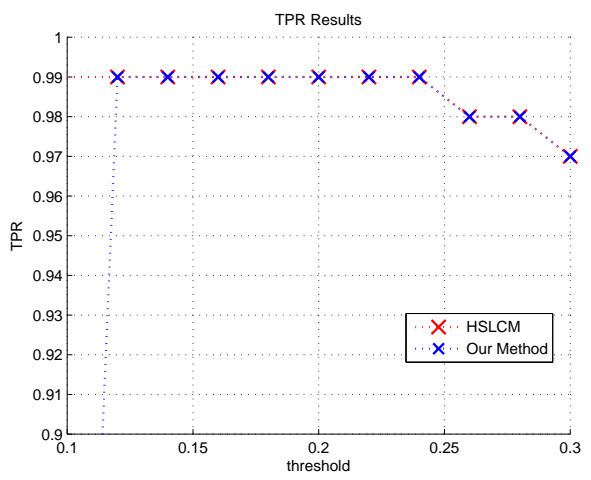

(a)

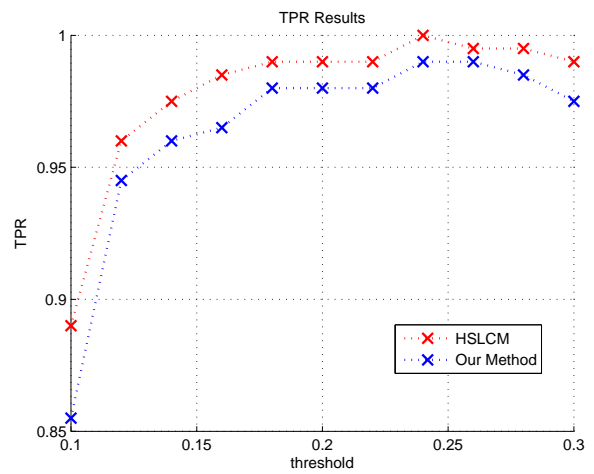

(b)

Figure 16: comparison of two methods' TPR.(a)TPR for circular target. (b)TPR for elliptic target.

In Fig.15, we can see that in most cases, the proposed algorithm can achieve the lowest FPR for a same threshold. Especially the threshold value set to 0.22 or higher, the FPR of our algorithm is less than ten percent.

From the Fig.16, we can get the conclusion that SVM classifier may reduce the detection rate of the algorithm, but still higher than other algorithms. This is determined by the classifier's own accuracy.

Finally, we compare the average computational cost of these detection methods for 100 images (see Fig.17). From the experimental results, we can get the conclusion that top-hat method has the fastest computing speed in these four methods. The proposed algorithm is just a few seconds slower than TDLMS algorithm. The method of sliding window of LCM method based on pixel mobile makes its calculation speed slowest.

We choose 0.2 as threshold to compare the performance of all methods and list performance index in table 1(cir- 


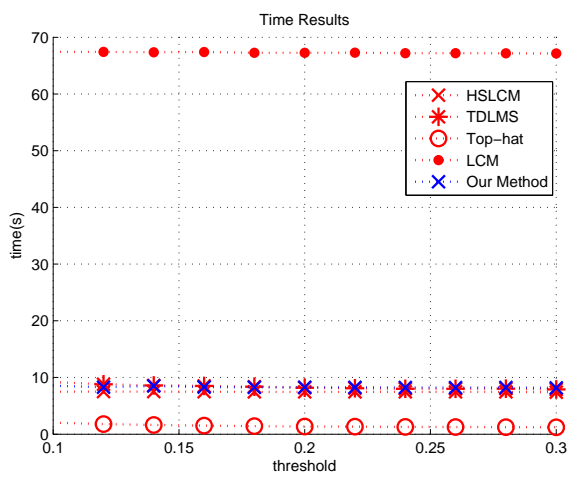

(a)

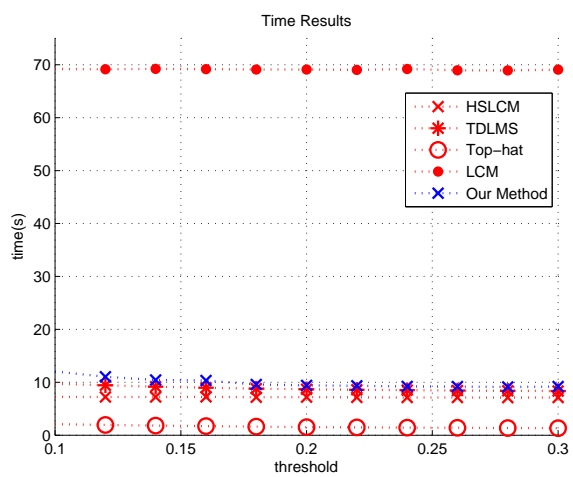

(b)

Figure 17: comparison of several methods' time.(a)time for circular target. (b)time for elliptic target.

cular target) and table 2(elliptic target). We mainly analyze the elliptic target. From the experimental results, we can get the conclusion that HSLCM algorithm has a 8 percent increase on TPR, but a seventh of operation time. After get additional SVM classifier, we reduce three times of FPR despite 1 percent of reduce of TPR. In general we can get the conclusion that top-hat method and TDLMS algorithm can achieve fast detection speed; however, their FPR may be a little high. LCM algorithm can achieve better performance in TPR, but its detection speed is limited. The proposed algorithm has a same detection speed with TDLMS algorithm but also the best performance in FPR in all methods.

\begin{tabular}{lccc} 
Table 1: Result of all methods for circular target $(\mathrm{K}=0.2)$ \\
Method & TPR & FPR & time \\
\hline HSLCM+SVM & 0.99 & 0.0196 & 8.284 \\
HSLCM & 0.99 & 0.9132 & 7.472 \\
TOP-HAT & 0.99 & 0.9303 & 1.357 \\
TDLMS & 0.99 & 0.9339 & 8.19 \\
LCM & 0.99 & 0.9199 & 67.268 \\
\hline
\end{tabular}

The result of the experiment shows that traditional algorithm has a big difficulty in detecting small target in complex background on the ground. The proposed algorithm can greatly reduce the FPR.

\section{CONCLUSION}

In this paper, an effective small target detection algorithm motivated by HVS is presented. The key idea of the proposed method is to use the HSLCM to enhance the targets and use a threshold operation to choose the most significant areas. Then SVM classifier separates targets from background clutters. Experimental results show that the 
Table 2: Result of all methods for elliptic target $(\mathrm{K}=0.2)$.

\begin{tabular}{lccc}
\hline Method & TPR & FPR & time \\
\hline HSLCM+SVM & 0.98 & 0.2248 & 9.704 \\
HSLCM & 0.99 & 0.7375 & 7.191 \\
TOP-HAT & 0.9 & 0.8686 & 1.529 \\
TDLMS & 0.905 & 0.9019 & 8.672 \\
LCM & 0.975 & 0.7899 & 69.108 \\
\hline
\end{tabular}

proposed algorithm is robust to resist pseudo targets and can achieve a high detection rate in less than $0.1 \mathrm{~s}$ with a fast calculation speed. It is worth noting that the FPR of proposed algorithm is far below other algorithms. This algorithm can be either directly used in single-frame target detection or used as a foundation module in sequential target tracking for real-time applications.

Based on the above conclusions, the proposed algorithm has a balanced performance on TPR, FPR and detection time.

\section{Acknowledgement}

This work is supported by the Fundamental Research Funds for the Central Universities(Grant No.HIT.NSRIF.2017014)

\section{References}

[1] G. Wen-pu, S. Ji-yin, L. Hao, Dynamic template preparing and application on flir target recognition, in: Electrical and Control Engineering (ICECE), 2010 International Conference on, IEEE, 2010, pp. 864-867.

[2] V. T. Tom, T. Peli, M. Leung, J. E. Bondaryk, Morphology-based algorithm for point target detection in infrared backgrounds, in: Optical Engineering and Photonics in Aerospace Sensing, International Society for Optics and Photonics, 1993, pp. 2-11.

[3] S. D. Deshpande, H. E. Meng, R. Venkateswarlu, P. Chan, Max-mean and max-median filters for detection of small targets, in: SPIE's International Symposium on Optical Science, Engineering, and Instrumentation, International Society for Optics and Photonics, 1999, pp. $74-83$.

[4] P. Wang, J. Tian, C. Q. Gao, Infrared small target detection using directional highpass filters based on 1s-svm, Electronics letters 45 (3) (2009) $156-158$.

[5] Y. Gu, C. Wang, B. Liu, Y. Zhang, A kernel-based nonparametric regression method for clutter removal in infrared small-target detection applications, Geoscience and Remote Sensing Letters, IEEE 7 (3) (2010) 469-473.

[6] K. E. Matthews, N. M. Namazi, A bayes decision test for detecting uncovered-background and moving pixels in image sequences., IEEE Transactions on Image Processing 7 (5) (1998) 720-728.

[7] M. M. Hadhoud, D. W. Thomas, The two-dimensional adaptive lms (tdlms) algorithm, Circuits and Systems, IEEE Transactions on 35 (5) (1988) 485-494.

[8] D.-J. Guo, Z.-M. Lu, H. Luo, Multi-channel adaptive mixture background model for real-time tracking, source: Journal of Information Hiding and Multimedia Signal Processing 7 (1) (2016) 216-221. 
[9] X. Hou, L. Zhang, Saliency detection: A spectral residual approach, in: Computer Vision and Pattern Recognition, 2007. CVPR'07. IEEE Conference on, IEEE, 2007, pp. 1-8.

[10] C. Guo, Q. Ma, L. Zhang, Spatio-temporal saliency detection using phase spectrum of quaternion fourier transform, in: Computer vision and pattern recognition, 2008. cvpr 2008. ieee conference on, IEEE, 2008, pp. 1-8.

[11] J. Li, M. D. Levine, X. An, X. Xu, H. He, Visual saliency based on scale-space analysis in the frequency domain, Pattern Analysis and Machine Intelligence, IEEE Transactions on 35 (4) (2013) 996-1010.

[12] M.-Y. Fang, C.-K. Chang, N.-C. Yang, C.-M. Kuo, S.-K. Guang, et al., Robust player tracking for broadcast tennis videos with adaptive kalman filtering, source: Journal of Information Hiding and Multimedia Signal Processing 5 (2) (2014) $242-262$.

[13] S. Kim, Y. Yang, J. Lee, Y. Park, Small target detection utilizing robust methods of the human visual system for irst, Journal of Infrared Millimeter and Terahertz Waves 30 (9) (2009) 994-1011.

[14] J. Li, D. Shi, W. Yang, Infared target extraction in flir imagery based on spatio temporal using fuzzy clustering, in: Intelligent Information Technology Application, 2008. IITA'08. Second International Symposium on, Vol. 1, IEEE, 2008, pp. 848-851.

[15] C. Gao, D. Meng, Y. Yang, Y. Wang, X. Zhou, A. G. Hauptmann, Infrared patch-image model for small target detection in a single image, Image Processing, IEEE Transactions on 22 (12) (2013) 4996-5009.

[16] S. Zheng, J. Liu, J.-W. Tian, An svm-based small target segmentation and clustering approach, in: Machine Learning and Cybernetics, 2004. Proceedings of 2004 International Conference on, Vol. 6, IEEE, 2004, pp. 3318-3323.

[17] C. Cortes, V. Vapnik, Support-vector networks, Machine learning 20 (3) (1995) 273-297.

[18] J. Davis, M. Goadrich, The relationship between precision-recall and roc curves, in: Proceedings of the 23rd international conference on Machine learning, ACM, 2006, pp. 233-240. 